\title{
SPECTRAL DECOMPOSITIONS IN BANACH SPACES AND THE HILBERT TRANSFORM
}

\author{
T. A. GILLESPIE \\ Department of Mathematics and Statistics \\ University of Edinburgh, James Clerk Maxwell Building \\ Edinburgh EH9 3JZ, Scotland \\ E-mail: t.a.gillespie@edinburgh.ac.uk
}

\begin{abstract}
This paper gives a survey of some recent developments in the spectral theory of linear operators on Banach spaces in which the Hilbert transform and its abstract analogues play a fundamental role.

1. Introduction. Various notions of self-adjointness have been developed for operators acting on Banach spaces, each reflecting some aspect of the Hilbert space theory. One such notion is that of well-boundedness, a concept introduced by Smart [23] and first studied by Smart and Ringrose [21-23]. An operator is (by definition) well-bounded if it has a functional calculus based on the Banach algebra of absolutely continuous functions on a compact real interval. This functional calculus gives rise to a form of spectral diagonalization, as will be described in more detail below.

Initially, there were relatively few examples of well-bounded operators, other than rather obvious ones, until Dowson and Spain [17] gave an interesting example of a wellbounded operator $A$ acting on $L^{p}(\mathbb{Z})$ for $p$ in the range $1<p<\infty$. It can be shown that the operator they considered has the property that $e^{i A}$ is the bilateral shift on $L^{p}(\mathbb{Z})$ (see [18, p. 1044]) and this observation illustrates the more general fact [19] that every translation operator on $L^{p}(G)$, where $G$ is a locally compact abelian group and $1<p<\infty$, is of the form $e^{i A}$ for some well-bounded operator $A$.

These examples suggest that the "unitary" concept corresponding to well-boundedness might be worthy of consideration and this was taken up in [2], where the concept of a trigonometrically well-bounded operator was introduced and studied systematically. Soon after, it was shown in [4] that every power-bounded invertible operator acting on a closed subspace of an arbitrary $L^{p}$ space $(1<p<\infty)$ is trigonometrically well-bounded and so has a spectral diagonalization similar to, although in general weaker than, a unitary
\end{abstract}

1991 Mathematics Subject Classification: 47B40, 42A50.

The paper is in final form and no version of it will be published elsewhere. 
operator on a Hilbert space. This, incidentally, puts the above result about translation operators on $L^{p}(G)$ into a much more general context.

The aim of the present paper is to give a fairly informal account of how the theory of trigonometrically well-bounded operators has developed over the past decade and to discuss the crucial role played by the Hilbert transform and its abstract analogues in this development. To put matters into a historical context, the paper starts with a discussion of how the theory emerged naturally from the earlier work of Dunford and his school on spectral operators.

Throughout, all Banach spaces will be over the complex scalars $\mathbb{C}$. Given a Banach space $X, X^{*}$ will denote its dual space and $L(X)$ the Banach algebra of all bounded linear operators on $X$. The identity operator on $X$ will be denoted by $I$ and the spectrum of $T \in L(X)$ by $\sigma(T)$. As usual, $\mathbb{R}, \mathbb{Z}$ and $\mathbb{T}$ will denote the reals, the integers and the circle group respectively. Given a compact interval $J=[a, b]$ in $\mathbb{R}, A C(J)$ will denote the Banach algebra of all absolutely continuous functions $f: J \rightarrow \mathbb{C}$, with norm

$$
\|f\|_{J}=|f(b)|+\operatorname{var}_{J} f .
$$

The Banach algebra of all functions $f: \mathbb{T} \rightarrow C$ of bounded variation with norm

$$
\|f\|_{\mathbb{T}}=|f(1)|+\operatorname{var}_{\mathbb{T}} f
$$

will be denoted by $B V(\mathbb{T})$ and the subalgebra of absolutely continuous complex valued functions on $\mathbb{T}$ by $A C(\mathbb{T})$.

2. Self-adjoint and unitary operators on Banach spaces. The spectral theorem for a normal operator $N$ on a Hilbert space $H$ gives an infinite dimensional diagonal representation of $N$ in terms of a spectral integral. More precisely, $N$ can written as

$$
N=\int_{\sigma(N)} z \mathcal{E}(d z)
$$

where $\mathcal{E}(\cdot)$ is the spectral measure of $N$, a self-adjoint projection-valued function defined on the Borel subsets of $\sigma(N)$ which is countably additive in the strong operator topology. The integral is obtained in a natural way by approximating the integrand uniformly on $\sigma(N)$ by simple Borel functions, the resulting integral (2.1) then being defined as a limit with respect to the operator norm.

Motivated in part by this and in part by the Jordan canonical form for a matrix, N. Dunford introduced the notion of a scalar-type spectral operator on a Banach space. This is an operator $S$ which can be written as

$$
S=\int_{\sigma(S)} z \mathcal{E}(d z),
$$

where $\mathcal{E}(\cdot)$ is a projection-valued measure defined, as before, on the Borel subsets of $\sigma(S)$. There is, however, a crucial difference between the Hilbert and Banach space settings. For a normal operator $N$, the existence of the corresponding spectral measure is a consequence of the commutativity of $N$ and $N^{*}$ whereas the Dunford theory requires that the existence of $\mathcal{E}(\cdot)$ be postulated at the outset. To be sure, sufficient conditions on $S$ for the existence of $\mathcal{E}(\cdot)$ can be given in certain situations, but there is nothing quite as simple as the 
normality condition $N N^{*}=N^{*} N$, which is both a necessary and a sufficient condition for a representation of the form (2.1) in the Hilbert space setting.

Another feature of the Banach space theory is that the countable additivity condition on $\mathcal{E}(\cdot)$ is relatively restrictive and, as a consequence, many operators on classical Banach spaces do not fall within the framework of the theory of spectral operators. It would be desirable, therefore, to weaken the integral representations of the form (2.1) in the hope of developing a theory which will apply to a larger class of operators. One way to attempt this is to consider an operator $S$ with real spectrum (corresponding, of course, to the self-adjoint case in the Hilbert space setting).

Accordingly, let $A$ be a scalar-type spectral operator with real spectrum contained in some closed interval $[a, b]$ and spectral measure $\mathcal{E}(\cdot)$. We may extend $\mathcal{E}(\cdot)$ to the whole of $\mathbb{R}$ by defining it to be zero on Borel subsets of $\mathbb{R} \backslash \sigma(A)$. Let $E(\lambda)=\mathcal{E}((-\infty, \lambda])$ for $\lambda \in \mathbb{R}$. We have

$$
A=\int_{[a, b]} \lambda \mathcal{E}(d \lambda)
$$

and this can be rewritten as

$$
A=a E(a)+\int_{[a, b]} \lambda d E(\lambda)
$$

the term $a E(a)$ being included to take account of the possibility that $\mathcal{E}(\cdot)$ may have some mass at the singleton $\{a\}$. The integral in (2.2) exists as a Riemann-Stieltjes integral in the strong operator topology. Notice that the right-hand side of (2.2) makes sense for a wider class of projection-valued functions $E(\cdot)$ than those arising from a spectral measure.

Define a spectral family in a Banach space $X$ to be a projection-valued function $E(\cdot): \mathbb{R} \rightarrow L(X)$ with the following properties:

(i) $\sup \{\|E(\lambda)\|: \lambda \in \mathbb{R}\}<\infty$;

(ii) $E(\lambda) E(\mu)=E(\mu) E(\lambda)=E(\lambda)$ if $-\infty<\lambda \leq \mu<\infty$;

(iii) $E(\mu) x \rightarrow E(\lambda) x$ in norm as $\mu \rightarrow \lambda+$ for each $x \in X$;

(iv) for each $x \in X$, the left-hand limit $E\left(\lambda^{-}\right) x$ exists in the norm topology;

(v) for each $x \in X, E(\lambda) x \rightarrow x$ as $\lambda \rightarrow \infty$ and $E(\lambda) x \rightarrow 0$ as $\lambda \rightarrow-\infty$, both limits being in the norm topology.

Informally, a spectral family is a projection-valued function defined on $\mathbb{R}$ which increases from 0 to $I$ and has certain one-sided continuity properties relative to the strong operator topology. Notice that, when $X$ is reflexive, property (iv) follows from the remaining properties, whilst (i) follows from (ii) $-(\mathrm{v})$ in a general Banach space. If (v) is strengthened to the condition

$\left(\mathrm{v}^{\prime}\right)$ there exist $a, b \in \mathbb{R}$ such that $E(\lambda)=0$ for $\lambda<a$ and $E(\lambda)=I$ for $\lambda \geq b$, we say that $E(\cdot)$ is concentrated on $[a, b]$.

Given a spectral family in $X$ which is concentrated on the interval $J=[a, b]$ and $f \in A C(J)$, the integral

$$
\int_{J} f(\lambda) d E(\lambda)
$$


exists in the strong operator topology as a Riemann-Stieltjes integral. Furthermore, properties (ii) and $\left(\mathrm{v}^{\prime}\right)$ imply that the mapping $\Phi: A C(J) \rightarrow L(X)$ defined by

$$
\Phi(f)=f(a) E(a)+\int_{J} f(\lambda) d E(\lambda)
$$

is an identity-preserving algebra homomorphism, and integration by parts in (2.3) leads to the inequality

$$
\|\Phi(f)\| \leq K\|f\|_{J} \quad \text { for } f \in A C(J)
$$

where $K \equiv \sup \{\|E(\lambda)\|: \lambda \in J\}$. For brevity, the right-hand side of (2.3) is usually denoted by

$$
\int_{J}^{\oplus} f(\lambda) d E(\lambda)
$$

In particular, if

$$
A=\int_{J}^{\oplus} \lambda d E(\lambda)
$$

and $p$ is a complex polynomial, then $p(A)=\Phi(p)$ and

$$
\|p(A)\| \leq K\|p\|_{J} .
$$

Following the terminology of Smart [23], we say that a bounded linear operator $A$ on a Banach space $X$ is well-bounded if there is a compact interval $J$ in $\mathbb{R}$ and a constant $K$ such that (2.5) holds for all polynomials $p$. The above discussion shows that, if $A$ has a representation of the form $(2.4)$ for some spectral family $E(\cdot)$ in $X$, then $A$ is well-bounded. When $X$ is reflexive, the converse statement holds [21, 23].

TheOrem 1. Let $X$ be a reflexive Banach space and let $A \in L(X)$. Then $A$ is wellbounded if and only if there is a spectral family $E(\cdot)$ in $X$, concentrated on a compact interval $J$ in $\mathbb{R}$, such that

$$
A=\int_{J}^{\oplus} \lambda d E(\lambda) .
$$

When $A$ is well-bounded, the spectral family $E(\cdot)$ is uniquely determined (and is called the spectral family of $A$ ).

There is a version of Theorem 1 valid in the non-reflexive case [22] but it involves a weaker notion of spectral family involving projections acting on $X^{*}$ and the spectral integrals are interpreted in a weak-star sense. (See also $[14,15]$ for a slightly different approach.) Furthermore, the uniqueness assertion is no longer valid. In the present paper, however, we shall mainly consider the reflexive case since the theory is more elegant in that context and encompasses the main examples.

Well-boundedness gives an analogue on Banach spaces of the Hilbert space concept of self-adjointness and suggests a corresponding analogue for the class of unitary operators. An operator $U$ on a Hilbert space $H$ is unitary if and only if it can be written as

$$
U=\int_{\mathbb{T}} \omega \mathcal{E}(d \omega)
$$


for some self-adjoint spectral measure $\mathcal{E}(\cdot)$ on the Borel subsets of the unit circle. With $U$ represented in this way, let $E(\lambda)=\mathcal{E}\left(\Gamma_{\lambda}\right)$ for $0 \leq \lambda \leq 2 \pi$, where $\Gamma_{\lambda}$ is the arc $\left\{e^{i \lambda}: 0 \leq t \leq \lambda\right\}$, and extend $E(\cdot)$ to $\mathbb{R}$ by setting

$$
E(\lambda)=0 \quad(\lambda<0), \quad E(\lambda)=I \quad(\lambda \geq 2 \pi) .
$$

We obtain a spectral family in $H$ concentrated on $[0,2 \pi]$ and (2.6) can be rewritten as

$$
U=\int_{[0,2 \pi]}^{\oplus} e^{i \lambda} d E(\lambda) .
$$

This leads us to consider an operator $U$ on a Banach space $X$ which has a representation of the form (2.7) for some spectral family $E(\cdot)$ in $X$ concentrated on $[0,2 \pi]$. Such an operator $U$ is invertible with inverse $\int_{[0,2 \pi]}^{\oplus} e^{-i \lambda} d E(\lambda)$ and the mapping $f \rightarrow \int_{[0,2 \pi]}^{\oplus} f\left(e^{i \lambda}\right) d E(\lambda)$ of $A C(\mathbb{T})$ into $L(X)$ is a norm-continuous, identity-preserving algebra homomorphism. In particular, writing $q(U)$ for $\sum_{n \in \mathbb{Z}} a_{n} U^{n}$, where $q\left(e^{i t}\right)=\sum_{n \in \mathbb{Z}} a_{n} e^{i n t}$ is a trigonometric polynomial, we have

$$
\|q(U)\| \leq K\|q\|_{\mathbb{T}},
$$

where $K \equiv \sup \{\|E(\lambda)\|: \lambda \in[0,2 \pi]\}$. This is the analogue of (2.5) in the present situation.

In view of the terminology for well-bounded operators, we shall say that an operator $U$ on a Banach space $X$ is trigonometrically well-bounded if it is invertible and there is a constant $K$ such that (2.8) holds for all trigonometric polynomials. The above discussion shows that every operator $U$ with a representation as in (2.7) is trigonometrically wellbounded. Strictly speaking, the existence of such a representation was originally taken as the definition of trigonometrical well-boundedness [2], although the present definition is more natural, given the definition of well-boundedness. However, the two definitions coincide on reflexive spaces.

Theorem $2([2, \S 2]$ and $[1, \S 3])$. Let $X$ be a reflexive Banach space and let $U \in L(X)$. Then $U$ is trigonometrically well-bounded if and only if there is a spectral family $E(\cdot)$ in $X$ concentrated on $[0,2 \pi]$ such that

$$
U=\int_{[0,2 \pi]}^{\oplus} e^{i \lambda} d E(\lambda)
$$

When $U$ is trigonometrically well-bounded, the spectral family $E(\cdot)$ can be chosen to be left continuous in the strong operator topology at $2 \pi$ and, with this normalization, is uniquely determined. (It is then called the spectral decomposition of $U$.)

Corresponding to the fact that an operator on a Hilbert space is unitary if and only if is of the form $e^{i A}$ for some self-adjoint $A$, we have the corresponding connection between well-bounded and trigonometrically well-bounded operators.

Theorem 3 ([2, Corollary 2.17 and Proposition 3.1]). An operator $U$ on a reflexive Banach space $X$ is trigonometrically well-bounded if and only if it is of the form $e^{i A}$ for some well-bounded operator $A$ on $X$. In this case, $A$ can be chosen to have spectrum 
contained in $[0,2 \pi]$ with $2 \pi$ not an eigenvalue and these spectral conditions determine $A$ uniquely. ( $A$ is then called the argument of $U$ and is denoted by $\arg U$.)

It is easy to see that, if $U$ is trigonometrically well-bounded with spectral decomposition $E(\cdot)$, then $\arg U=\int_{[0,2 \pi]}^{\oplus} \lambda d E(\lambda)$.

3. An example. An instructive example of a trigonometrically well-bounded operator is provided by the bilateral shift $U$ on the space $L^{p}(\mathbb{Z})$ for $p$ in the range $1<p<\infty$. The operator $U$ is unitary on $L^{2}(\mathbb{Z})$ and an invertible isometry on $L^{p}(\mathbb{Z})$ for $1 \leq p \leq \infty$. It is easy to check that, given a trigonometric polynomial $q\left(e^{i t}\right)=\sum a_{n} e^{i n t}$, the operator $q(U)$ is given by convolution by the sequence $\left\{a_{n}\right\}$. Note that there are no convergence problems here since $\left\{a_{n}\right\}$ has finite support. It follows that, when acting on $L^{1}(\mathbb{Z})$ or $L^{\infty}(\mathbb{Z}),\|q(U)\|=\sum\left|a_{n}\right|$ and hence that there is no inequality of the form $\|q(U)\| \leq$ $K\|q\|_{\mathbb{T}}$ valid for all trigonometric polynomials $q$ when the operator norm is computed on $L^{1}(\mathbb{Z})$ or $L^{\infty}(\mathbb{Z})$. For this would imply that every function in $A C(\mathbb{T})$ would have an absolutely convergent Fourier series and this is known not to be the case $[26,(3.7)$, p. 241]. Thus $U$ is certainly not trigonometrically well-bounded on $L^{1}(\mathbb{Z})$ or $L^{\infty}(\mathbb{Z})$.

The situation when $1<p<\infty$ is, however, very different. Here $U$ is trigonometrically well-bounded. An easy way to see this is to apply a theorem of S. B. Stečkin [24] which asserts that, for $p$ in the range $1<p<\infty$, there is a constant $C_{p}$ with the property that, for each trigonometric polynomial $q$, the norm on $L^{p}(\mathbb{Z})$ of convolution by the sequence $\{\widehat{q}(n)\}$ of Fourier coefficients of $q$ does not exceed $C_{p}\|q\|_{\mathbb{T}}$. Thus, taking operator norms on $L^{p}(\mathbb{Z})$ for $1<p<\infty,\|q(U)\| \leq C_{p}\|q\|_{\mathbb{T}}$ for all trigonometric polynomials and so $U$ is trigonometrically well-bounded. In other words, a vestige of the unitary spectral structure remains when we pass from the Hilbert space $L^{2}(\mathbb{Z})$ to the reflexive $L^{p}(\mathbb{Z})$ spaces.

The result of Stečkin which underlies the trigonometric well-boundedness of $U$ on the reflexive $L^{p}(\mathbb{Z})$ spaces follows by an integration-by-parts argument from the boundedness on $L^{p}(\mathbb{Z})$ when $1<p<\infty$ of convolution by the discrete Hilbert kernel

$$
h(n)=n^{-1} \quad(n \neq 0), \quad h(0)=0,
$$

that is, the discrete Hilbert transform $H_{\mathbb{Z}}$. This result is usually attributed to Titchmarsh [25], although it really goes back to the work of M. Riesz on the $L^{p}$-boundedness of the Hilbert transform on $\mathbb{T}$ and $\mathbb{R}$. (An interesting account of these matters and related correspondence between G. H. Hardy and M. Riesz is given by M. L. Cartwright [12].) The spectral decomposition and argument of $U$ can also be given in terms if $H_{\mathbb{Z}}$ and its conjugates as follows.

For $0<\lambda<2 \pi$, let $\chi_{\lambda}$ denote the characteristic function of the arc $\left\{e^{i t}: 2 \pi-\lambda \leq\right.$ $t \leq 2 \pi\}$ of $\mathbb{T}$. Then $\chi_{\lambda}$ has Fourier coefficients $\widehat{\chi}_{\lambda}(n)$ given by

$$
\widehat{\chi}_{\lambda}(n)=\frac{i}{2 \pi n}\left(1-e^{i n \lambda}\right) \quad(n \neq 0), \quad \widehat{\chi}_{\lambda}(0)=\frac{\lambda}{2 \pi} .
$$

It follows from the $L^{p}$-boundedness of $H_{\mathbb{Z}}$ that convolution by the sequence $\left\{\widehat{\chi}_{\lambda}(-n)\right\}$ on $L^{p}(\mathbb{Z})$ is also bounded when $1<p<\infty$. Indeed, if we denote this convolution operator by $E(\lambda)$, then

$$
E(\lambda)=\frac{\lambda}{2 \pi} I+\frac{i}{2 \pi}\left(V_{\lambda}^{-1} H_{\mathbb{Z}} V_{\lambda}-H_{\mathbb{Z}}\right)
$$


for $0 \leq \lambda \leq 2 \pi$, where $V_{\lambda}\left(\left\{x_{n}\right\}\right)=\left\{e^{i n \lambda} x_{n}\right\}$. Furthermore, it follows from (3.1) that

$$
\sup \{\|E(\cdot)\|: 0 \leq \lambda \leq 2 \pi\}<\infty \text {. }
$$

Also, we have $(E(\lambda) x)^{\wedge}=\chi_{\lambda} \cdot \widehat{x}$ (at least for a finitely supported sequence $x$ ), where - denotes the Fourier transform on $L^{p}(\mathbb{Z}) \cap L^{2}(\mathbb{Z})$. Hence each $E(\lambda)$ is a projection. If we define

$$
E(\lambda)=I \quad(\lambda \geq 2 \pi), \quad E(\lambda)=0 \quad(\lambda<0),
$$

it is readily verified that we obtain a spectral family concentrated on $[0,2 \pi]$ and that the corresponding well-bounded operator

$$
A=\int_{[0,2 \pi]}^{\oplus} \lambda d E(\lambda)
$$

is the operator on $L^{p}(\mathbb{Z})$ corresponding to the $p$-multiplier on $\mathbb{T}$ defined by $\varphi\left(e^{i t}\right)=$ $2 \pi-t(0 \leq t<2 \pi)$. Hence $\left(e^{i A} x\right)^{\uparrow}\left(e^{i t}\right)=e^{-i t} \widehat{x}\left(e^{i t}\right)$, again for finitely supported sequences $x$, from which it is seen that $e^{i A}=U$. The spectral family $E(\cdot)$ is continuous in the strong operator topology, so that $A$ has no eigenvalues. Thus $A=\arg U$. Since $\widehat{\varphi}(-n)=i n^{-1}$ for $n \neq 0$ and $\widehat{\varphi}(0)=\pi$, it follows that

$$
A=\pi I+i H_{\mathbb{Z}}
$$

The well-boundedness of $\pi I+i H_{\mathbb{Z}}$ was first observed in [17], although the connection with the bilateral shift was not noted there.

4. The ergodic Hilbert transform and trigonometric well-boundedness. The calculations in the above example show that the trigonometric well-boundedness and spectral structure of the bilateral shift on $L^{p}(\mathbb{Z})$ for $1<p<\infty$ is intimately related to the discrete Hilbert transform. In fact, this reflects the role of the Hilbert transform in the general theory of trigonometrically well-bounded operators. We discuss this in the present section.

As remarked earlier, a trigonometrically well-bounded operator $U$ on a reflexive space $X$ has both a unique spectral decomposition $E(\cdot)$ and a unique argument $A$. In view of the above example, it is natural to ask how $E(\cdot)$ and $A$ can be obtained directly from $U$.

It can be shown that, given an arbitrary $\varphi \in B V(\mathbb{T})$, the integral

$$
\int_{[0,2 \pi]}^{\oplus} \varphi\left(e^{i \lambda}\right) d E(\lambda)
$$

exists as a Riemann-Stieltjes integral in the strong operator topology on $L(X)$ (see [16] or [4]) provided, in the approximating Riemann sums, evaluation of $\varphi$ is at the right-hand endpoint of each partitioning interval. Denoting the resulting operator by $S_{\varphi}$, it can be verified that the mapping $\varphi \rightarrow S_{\varphi}$ is a norm-continuous, identity-preserving homomorphism of $B V(\mathbb{T})$ into $L(X)$. Writing $\sum_{n \in \mathbb{Z}} \widehat{\varphi}(n) e^{i n t}$ for the Fourier series of $\varphi$ and recalling both that

$$
U^{n}=\int_{[0,2 \pi]}^{\oplus} e^{i n \lambda} d E(\lambda) \quad(n \in \mathbb{Z})
$$


and that $\sum_{n \in \mathbb{Z}} \widehat{\varphi}(n) e^{i n t}$ converges to $\widetilde{\varphi}\left(e^{i t}\right)$, where $\widetilde{\varphi}$ is the normalization of $\varphi$ defined by

$$
\widetilde{\varphi}\left(e^{i t}\right)=\frac{1}{2}\left\{\lim _{s \rightarrow t+} \varphi\left(e^{i s}\right)+\lim _{s \rightarrow t-} \varphi\left(e^{i s}\right)\right\},
$$

it seems likely that there is some connection between the (formal) series $\sum_{n \in \mathbb{Z}} \widehat{\varphi}(n) U^{n}$ and the operator $S_{\varphi}$. In fact, using routine properties of Fourier series, it can be shown [4, Theorem (3.10)] that the series $\sum_{n \in \mathbb{Z}} \widehat{\varphi}(n) U^{n}$ is $(\mathrm{C}, 1)$ summable to $S_{\tilde{\varphi}}$. Taking $\varphi\left(e^{i t}\right)=t$ for $0 \leq t<2 \pi$ and using the fact that

$$
A=\int_{[0,2 \pi]}^{\oplus} \lambda d E(\lambda)
$$

it is readily seen that

$$
A=\pi(I-E(0))+\sum_{n=-\infty}^{\infty} i n^{-1} U^{n}
$$

in the strong operator topology, where ${ }^{\prime}$ denotes the omission of the term $n=0$. (As usual, $(\mathrm{C}, 1)$ summability in this context refers to the convergence of the Cesàro means of balanced partial sums.) The (C,1) sum of the series in (4.1) can be viewed as the ergodic Hilbert transform $H_{U}$ of $U$ and, with this notation, we may write (4.1) as

$$
\arg U=\pi(I-E(0))+i H_{U}
$$

In much the same way, taking $\varphi$ to be the characteristic function of the arc $\left\{e^{i t}: 0 \leq t\right.$ $\leq \lambda\}$, we also obtain the formula

$$
2^{-1}\left(E\left(\lambda^{-}\right)+E(\lambda)\right)=2^{-1} E(0)+\frac{\lambda}{2 \pi} I+\frac{i}{2 \pi}\left(H_{U_{\lambda}}-H_{U}\right)
$$

for $0 \leq \lambda<2 \pi$, where $U_{\lambda}$ is the trigonometrically well-bounded operator $e^{-i \lambda} U$. Writing $G_{\lambda}$ for the operator $\frac{\lambda}{2 \pi} I+\frac{i}{2 \pi}\left(H_{U_{\lambda}}-H_{U}\right)$, we can use (4.3) to express $E(\lambda)$ directly in terms of the ergodic Hilbert transforms of $U$ and its rotates $U_{\lambda}$ as

$$
E(\lambda)=3 G_{\lambda}-2 G_{\lambda}^{2}
$$

for $\lambda$ in the range $0<\lambda<2 \pi$. Notice also that (4.3) implies that

$$
E(0)=\lim _{\lambda \rightarrow 0+} \frac{i}{\pi}\left(H_{U_{\lambda}}-H_{U}\right)
$$

in the strong operator topology. We now have, in (4.2), (4.4) and (4.5), expressions for the argument and spectral decomposition of a trigonometrically well-bounded operator $U$ on a reflexive space in terms of the ergodic Hilbert transform of $U$ and its rotates $U_{\lambda}$. In fact, these formulae will apply in the Banach space setting provided $U$ is assumed to be of the form

$$
U=\int_{[0,2 \pi]}^{\oplus} e^{i \lambda} d E(\lambda)
$$

for a normalized spectral family concentrated on $[0,2 \pi]$, but this is not guaranteed from (2.8) without reflexivity.

The formal ergodic Hilbert transform of the rotates of an invertible operator $U$ can also be used to characterise the trigonometrical well-boundedness of $U$. To make this 
more precise, let $\sigma_{n}\left(e^{i t}\right)$ be the $n$th Cesàro mean of the balanced partial sums of the trigonometric series

$$
\sum_{n=-\infty}^{\infty} \frac{e^{i n t}}{n}
$$

and let $U$ be an invertible operator on a Banach space $X$. Then $\left\{\sigma_{n}: n \in \mathbb{N}\right\}$ is bounded with respect to the $B V$ norm $\|\cdot\|_{\mathbb{T}}$ and hence, if $U$ satisfies (2.8) for all trigonometric polynomials $q$, then the sequence $\left\{\sigma_{n}(U): n \in \mathbb{N}\right\}$ is bounded in norm. Furthermore, if for a trigonometric polynomial $q$ and $\lambda \in \mathbb{R}, q_{\lambda}$ denotes the rotated polynomial $q_{\lambda}\left(e^{i t}\right)=$ $q\left(e^{i \lambda} e^{i t}\right)$, then $\left\|q_{\lambda}\right\|_{\mathbb{T}} \leq 2\|q\|_{\mathbb{T}}$. It follows immediately that, if $U$ satisfies (2.8), then there is a constant $M$ such that

$$
\left\|\sigma_{n}\left(U_{\lambda}\right)\right\| \leq M
$$

for all $\lambda \in \mathbb{R}$ and all $n \in \mathbb{N}$, where $U_{\lambda}=e^{i \lambda} U$. In other words, the Cesàro means $\left\{\sigma_{n}\left(U, e^{i \lambda}\right)\right\}$ of the partial sums of the trigonometric series

$$
\sum_{n=-\infty}^{\infty} \frac{e^{i n \lambda}}{n} U^{n}
$$

are uniformly bounded for $e^{i \lambda} \in \mathbb{T}$. The series (4.6) is, of course, the formal ergodic Hilbert transform of $U_{\lambda}$.

Conversely, suppose that there is a constant $M$ such that

$$
\left\|\sigma_{n}\left(U, e^{i \lambda}\right)\right\| \leq M
$$

for all $n \in \mathbb{N}$ and all $e^{i \lambda} \in \mathbb{T}$ and let $q$ be a trigonometric polynomial of degree $N$. For $m \geq N$, we have

$$
\begin{aligned}
q(U) & =\widehat{q}(0) I+\sum_{n=-m}^{m} \frac{1}{2 \pi} \int_{0}^{2 \pi} q\left(e^{i \lambda}\right) e^{-i n \lambda} U^{n} d \lambda \\
& =\widehat{q}(0) I-\frac{i}{2 \pi} \int_{0}^{2 \pi}\left\{\sum_{n=-m}^{m} e^{-i n \lambda} \frac{U^{n}}{n}\right\} d q\left(e^{i \lambda}\right) .
\end{aligned}
$$

Since the terms in these sums are in fact zero for $|n|>N$, it is readily seen that

$$
q(U)-\widehat{q}(0) I+\frac{i}{2 \pi} \int_{0}^{2 \pi} \sigma_{n}\left(U, e^{-i \lambda}\right) d q\left(e^{i \lambda}\right) \rightarrow 0
$$

in norm as $n \rightarrow \infty$, from which it follows that $\|q(U)\| \leq\left(1+\frac{M}{2 \pi}\right)\|q\|_{\mathbb{T}}$.

We can thus summarise the above discussion in the following result.

THEOREM 4. Let $U$ be an invertible operator on a Banach space $X$. Then there exists a constant $K$ such that $\|q(U)\| \leq K\|q\|_{\mathbb{T}}$ for all trigonometric polynomials $q$ if and only if

$$
\sup \left\{\left\|\sigma_{n}\left(U, e^{i \lambda}\right)\right\|: n \in \mathbb{N} \text { and } \lambda \in \mathbb{R}\right\}<\infty .
$$

When $X$ is reflexive, (4.7) can be replaced by the stronger condition that $\left\{\sigma_{n}\left(U, e^{i \lambda}\right)\right\}$ converges in the strong (or weak) operator topology at each $e^{i \lambda} \in \mathbb{T}$ with limit which is uniformly bounded for $e^{i \lambda} \in \mathbb{T}$. This is discussed in [3] in greater detail. 
5. UMD spaces and trigonometric well-boundedness. The results of the previous section show that, in order to check the well-boundedness condition (2.8), it is sufficient (as well as necessary) to verify it only for the trigonometric polynomials $\sigma_{n}\left(e^{i t}\right)$ and their rotates. Whilst this is of theoretical interest, it may not be easy to do for a particular operator $U$. In this section, we show how to do so when $U$ is power-bounded and the underlying space belongs to a particular class of Banach spaces.

The class in question consists of the so-called UMD spaces. These are the spaces $X$ for which the Hilbert transform has the same boundedness properties when considered as acting on the Lebesgue-Bochner spaces of $X$-valued functions as the classical Hilbert transform on spaces of scalar-valued functions. To make this more precise, the formula

$$
\left(H_{\mathbb{R}, X} f\right)(t)=\frac{1}{\pi} \int_{-\infty}^{\infty} \frac{f(t-s)}{s} d s
$$

makes sense for almost all $t \in \mathbb{R}$ in the principal value sense for (say) all compactly supported $X$-valued simple functions $f$. One can then ask whether the resultant $X$ valued Hilbert transform $H_{\mathbb{R}, X}$ is bounded relative to the norm $\|\cdot\|_{p}$ inherited from the Lebesgue-Bochner space $L^{p}(\mathbb{R}, X)$ of all $p$-integrable $X$-valued functions on $\mathbb{R}$ when $p$ is in the range $1<p<\infty$, as in the scalar case. One can ask a similar questions of the discrete $X$-valued Hilbert transform $H_{\mathbb{Z}, X}$, that is convolution by the discrete Hilbert kernel $h$ on the space $L^{p}(\mathbb{Z}, X)$ of $p$-summable sequences of elements of $X$, and of the periodic Hilbert transform $H_{\mathbb{T}, X}$ given by convolution by the function $e^{i t} \rightarrow \frac{1}{2 \pi} \cot \frac{t}{2}(0<|t|<\pi)$ on $L^{p}(\mathbb{T}, X)$. It can be shown that, for a given Banach space $X$, the $L^{p}$-boundedness of any one of these operators for any single value of $p$ in the range $1<p<\infty$ ensures the $L^{p}$-boundedness of all three for every value of $p$ in this range. Furthermore, this is equivalent both to a geometric property of $X$ called $\zeta$-convexity and to the property of $X$-valued martingale difference sequences referred to as the unconditionality property for martingale differences. Banach spaces having these properties are called UMD spaces and are automatically reflexive. These results are due to Burkholder and Bourgain [11], [9] (see also [7] where the discrete Hilbert transform is considered and further background references are given).

To attack the problem of verifying the trigonometric well-boundedness property $(2.8)$ when $U$ is an invertible power-bounded operator (that is, $\sup \left\{\left\|U^{n}\right\|: n \in \mathbb{Z}\right\}<\infty$ ) we use a technique called transference. The background for this is as follows. Let $G$ be a locally compact abelian group and let $u \rightarrow R_{u}$ be a strongly continuous representation of $G$ in a Banach space $X$ such that

$$
c \equiv \sup \left\{\left\|R_{u}\right\|: u \in G\right\}<\infty .
$$

This gives rise to a representation $k \rightarrow T_{k}$ of the group algebra $L^{1}(G)$ by means of the formula

$$
T_{k}=\int_{G} k(u) R_{-u} d u,
$$

where integration is with respect to Haar measure on $G$ and the integral exists as a Bochner integral in the strong operator topology. The estimate $\left\|T_{k}\right\| \leq c\|k\|_{1}$ is immediate 
from (5.1). However, an averaging argument [8, Theorem (2.8)] gives the estimate

$$
\left\|T_{k}\right\| \leq c^{2} N_{p, X}(k),
$$

where $1 \leq p<\infty$ and $N_{p, X}(k)$ denotes the norm of convolution by $k$ on the LebesgueBochner space $L^{p}(G, X)$ of all $p$-integrable $X$-valued functions on $G$. Notice that, when $u \rightarrow R_{u}$ is the regular representation of $G$ in $L^{p}(X, G), T_{k}$ is given by convolution by $k$. The force of (5.2) is that, for an arbitary bounded representation, the norm of the transferred operator $T_{k}$ is controlled by the norm $N_{p, X}(k)$ of the transferred operator in this special case. The inequality (5.2), as well as its proof, is a mild adaptation of the transference result of $\mathrm{R}$. R. Coifman and G. Weiss [13, Theorem 2.4], in which $X$ is a subspace of an $L^{p}$-space of scalar-valued functions and $N_{p, X}(k)$ is replaced by the norm $N_{p}(k)$ of convolution by $k$ on $L^{p}(G)$. Note that, in the scalar case, a particular value of $p$ is specified at the outset, corresponding to the $L^{p}$-subspace in which $G$ is represented, and the estimate $\left\|T_{k}\right\| \leq c^{2} N_{p}(k)$ involves the same value of $p$. On the other hand, in the vector case no particular value of $p$ is specified in advance and an inequality of the form (5.2) is obtained for each value of $p$ in the range $1 \leq p<\infty$.

Suppose now that $U$ is an invertible operator on a UMD space $X$ with $c \equiv \sup \left\{\left\|U^{n}\right\|\right.$ : $n \in \mathbb{Z}\}<\infty$. This gives rise to the representation $n \rightarrow U^{n}$ of $\mathbb{Z}$ in $X$ and, given a trigonometric polynomial $q, q(U)$ is the transferred operator $T_{k}$ corresponding to the kernel $k$, where $k(n)=\widehat{q}(-n)=\check{q}(n)$ (here $\check{q}$ denotes the inverse Fourier transform of $q$ ). In particular, (5.2) gives the estimate

$$
\left\|\sigma_{n}(U)\right\| \leq c^{2} N_{p, X}\left(\check{\sigma}_{n}\right),
$$

where $\sigma_{n}$ is as in the previous section. For $1 \leq p<\infty$ and $\omega \in \mathbb{T}$, let $V_{\omega}$ be the invertible isometry on $L^{p}(\mathbb{Z}, X)$ defined by $V_{\omega}\left(\left\{x_{m}\right\}\right)=\left\{\omega^{m} x_{m}\right\}$. A simple calculation shows that, for $1<p<\infty$,

$$
\check{\sigma}_{n} * x=\int_{\mathbb{T}} \kappa_{n}(\omega)\left(V_{\omega} H_{\mathbb{Z}, X} V_{\omega}^{-1} x\right) d \omega \quad\left(x \in L^{p}(\mathbb{Z}, X)\right),
$$

where $H_{\mathbb{Z}, X}$ is the discrete Hilbert transform on $L^{p}(\mathbb{Z}, X)$ and $\kappa_{n}$ is the $n$th Fejér kernel. This leads to the estimate

$$
N_{p, X}\left(\check{\sigma}_{n}\right) \leq C_{p, X}
$$

for $n \in \mathbb{N}$, where $1<p<\infty$ and $C_{p, X}$ denotes the norm of the discrete Hilbert transform on $L^{p}(\mathbb{Z}, X)$. The UMD property for $X$ ensures that $C_{p, X}$ is finite. Using (5.3) and (5.4), it is seen that

$$
\left\|\sigma_{n}(U)\right\| \leq c^{2} C_{p, X}
$$

for $n \in \mathbb{N}$ and $1<p<\infty$. Replacing $U$ by $e^{i \lambda} U$, it follows that, for $1<p<\infty$,

$$
\left\|\sigma_{n}\left(U, e^{i \lambda}\right)\right\| \leq c^{2} C_{p, X}
$$

for all $e^{i \lambda} \in \mathbb{T}$ and all $n \in \mathbb{N}$. Hence, by Theorem $4, U$ is trigonometrically well-bounded.

Since spaces belonging to the class UMD are automatically reflexive, we can summarise what we have just proved in the following result. 
TheOREM 5 [7, Theorem 4.5]. Let $U$ be a power-bounded invertible operator on a UMD space $X$. Then there is a spectral family in $X$, concentrated on $[0,2 \pi]$, such that

$$
U=\int_{[0,2 \pi]}^{\oplus} e^{i \lambda} d E(\lambda)
$$

It is worth commenting that closed subspaces of $L^{p}$-spaces are UMD if $1<p<\infty$; in particular, Hilbert spaces belong to the class UMD. Since an invertible power-bounded operator on a Hilbert is similar to a unitary operator [16, Theorem 8.1], Theorem 5 shows that a vestige of this spectral structure remains for power-bounded operators on general UMD spaces.

Furthermore, it should be remarked that, although the spectral family in Theorem 5 will not in general generate a spectral measure on the Borel subsets of $[0,2 \pi]$, nevertheless it does give rise to a spectral measure associated with a dyadic partitioning of $[0,2 \pi]$ (see [6] for details). This can be viewed as an operator-theoretic analogue of the classical Littlewood-Paley theorem for $L^{p}(\mathbb{Z})$ and is underpinned by a version of the LittlewoodPaley result for $L^{p}(\mathbb{T}, X)$ valid when $X$ is a UMD space [10], together with several transference arguments.

6. Weighted shifts. We now turn to an analysis of weighted shift operators in the context of trigonometric well-boundedness. Since trigonometrically well-bounded operators are invertible, we consider a bilateral weighted shift acting on $L^{p}(\mathbb{Z})$; in other words, we analyse the structure of an operator $S_{\alpha}$ acting on $L^{p}(\mathbb{Z})$ of the form

$$
S_{\alpha}\left(\left\{x_{n}\right\}\right)=\left\{\alpha_{n} x_{n-1}\right\},
$$

where $\alpha=\left\{\alpha_{n}\right\}_{n \in \mathbb{Z}}$ is a bounded sequence. Since $S_{\alpha}$ is isometrically similar to $S_{\beta}$ where $\beta_{n}=\left|\alpha_{n}\right|$, we assume that $\alpha_{n} \geq 0$ for all $n$. Also, $S_{\alpha}$ is invertible if and only if the sequence $\left\{\alpha_{n}\right\}$ is bounded away from zero and so we must also assume this. To analyse $S_{\alpha}$, it is in fact convenient to analyse instead the unweighted shift

$$
U\left(\left\{x_{n}\right\}\right)=\left\{x_{n-1}\right\}
$$

acting on a weighted $L^{p}$ space. To make this more precise, set

$$
w_{n}= \begin{cases}\left(\alpha_{1} \ldots \alpha_{n}\right)^{p} & \text { if } n>0 \\ 1 & \text { if } n=0 \\ \left(\alpha_{n+1} \ldots \alpha_{0}\right)^{-p} & \text { if } n<0\end{cases}
$$

for $n \in \mathbb{Z}$, and let $L^{p}(\mathbb{Z}, w)$ denote the associated weighted $L^{p}$ space of complex sequences $x=\left\{x_{n}\right\}$ such that $\|x\|_{w, p} \equiv\left\{\sum_{n \in \mathbb{Z}}\left|x_{n}\right|^{p} w_{n}\right\}^{1 / p}<\infty$. It is straightforward to check that $S_{\alpha}$ acting on $L^{p}(\mathbb{Z})$ is isometrically similar to $U$ acting on $L^{p}(\mathbb{Z}, w)$. Conversely, starting with $U$ acting on a weighted $L^{p}$ space $L^{p}(\mathbb{Z}, w)$, where the weights $w_{n}$ are strictly positive, it is easy to construct a weighted shift $S_{\alpha}$ on $L^{p}(\mathbb{Z})$ isometrically similar to $U$. Thus the problem of analysing a weighted shift on $L^{p}(\mathbb{Z})$ is equivalent to the analysis of the unweighted shift $U$ on a weighted space $L^{p}(\mathbb{Z}, w)$. It is convenient to write $U_{w}$ to denote the unweighted shift $U$ when considered as acting on the weighted space $L^{p}(\mathbb{Z}, w)$. Notice that $U_{w}$ is bounded if and only of the sequence $\left\{w_{n+1} / w_{n}\right\}_{n \in \mathbb{Z}}$ is bounded, whilst 
$U_{w}$ is bounded and invertible if and only if $\left\{w_{n+1} / w_{n}\right\}_{n \in \mathbb{Z}}$ is bounded and bounded away from zero.

The criterion for trigonometric well-boundedness given by Theorem 4 takes on a simpler form in this situation since $U_{w}$ is isometrically similar to $e^{i \lambda} U_{w}$ for $\lambda \in \mathbb{R}$ whenever $U_{w}$ is bounded. Furthermore, given a trigonometric polynomial $q, q\left(U_{w}\right)$ is given by convolution by the sequence $\widehat{q}$. Thus, $U_{w}$ is a trigonometrically well-bounded operator on $L^{p}(\mathbb{Z}, w)$ if and only if it is bounded and invertible and there is a constant $K$ such that

$$
\left\|\widehat{\sigma}_{n} * x\right\|_{w, p} \leq K\|x\|_{w, p}
$$

for all finitely supported sequences $x$ and all $n \in \mathbb{N}$, where $\sigma_{n}$ is as in $\S 4$. It is not hard to show that $(6.2)$ is equivalent to the $L^{p}(\mathbb{Z}, w)$ boundedness of convolution by the discrete Hilbert kernel $h$. A deep result of Hunt, Muckenhoupt and Wheeden [20] gives the equivalence, for $1<p<\infty$, of the boundedness of the discrete Hilbert transform on $L^{p}(\mathbb{Z}, w)$ and the $A_{p}$-condition for the weight sequence $w$, namely that there is a constant $C$ such that

$$
\left\{\sum_{k \in I} w_{k}\right\}\left\{\sum_{k \in I} w_{k}^{-1 /(p-1)}\right\}^{p-1} \leq C|I|^{p}
$$

for all finite intervals $I$ in $\mathbb{Z}$, where $|I|$ denotes the cardinality of $I$. Furthermore, it is easy to verify that $U_{w}$ is bounded and invertible when $w$ satisfies the $A_{p}$-condition.

We thus have the following result (see [5, Theorem (4.2)]).

Theorem 6. Let $1<p<\infty$ and let $\alpha_{n} \in \mathbb{C}$ for $n \in \mathbb{Z}$. Then the operator $S_{\alpha}$ given by (6.1) is trigonometrically well-bounded on $L^{p}(\mathbb{Z})$ if and only if $\alpha_{n} \neq 0$ for all $n$ and the sequence $\left\{w_{n}\right\}_{n \in \mathbb{Z}}$ defined by

$$
w_{0}=1, \quad w_{n}=\left|\alpha_{1} \ldots \alpha_{n}\right|^{p} \quad \text { if } n>0, \quad \gamma_{n}=\left|\alpha_{n+1} \ldots \alpha_{0}\right|^{-p} \quad \text { if } n<0
$$

satisfies the $A_{p}$ condition.

This result gives an effective way of constructing examples to exhibit various aspects of the theory of trigonometrical well-boundedness. For instance, it can be used to show that there is no characterization of trigonometrical well-boundedness for an invertible operator $U$ on a Hilbert space in terms of the growth of $\left\|U^{n}\right\|$ as $|n| \rightarrow \infty$, contrasting with a result of J. Wermer [16, Theorem 8.3] that such an operator $U$ is a scalar-type spectral operator with spectrum contained in $\mathbb{T}$ if and only of $\left\|U^{n}\right\|=O(1)$ as $|n| \rightarrow \infty$ (see $[5, \S 5])$.

\section{References}

[1] H. Benzinger, E. Berkson and T. A. Gillespie, Spectral families of projections, semigroups, and differential operators, Trans. Amer. Math. Soc. 275 (1983), 431-475.

[2] E. Berkson and T. A. Gillespie, AC functions on the circle and spectral families, J. Operator Theory 13 (1985), 33-47.

[3] - - - Fourier series criteria for operator decomposability, Integral Equations Operator Theory 9 (1986), 767-789. 
[4] E. Berkson and T. A. Gillespie, Stečkin's theorem, transference, and spectral decompositions, J. Funct. Anal. 70 (1987), 140-170.

[5] - - - The spectral decomposition of weighted shifts and the $A_{p}$ condition, Colloq. Math. 60/61 (1990), 507-518.

[6] - - - Spectral decompositions and harmonic analysis on UMD spaces, Studia Math. 112 (1994), 13-49.

[7] E. Berkson, T. A. Gillespie and P. S. Muhly, Abstract spectral decompositions guaranteed by the Hilbert transform, Proc. London Math. Soc. (3) 53 (1986), 489-517.

[8] -, - - - Generalized analyticity in UMD spaces, Ark. Mat. 27 (1989), 1-14.

[9] J. Bourgain, Some remarks on Banach spaces in which martingale difference sequences are unconditional, ibid. 21 (1983), 163-168.

[10] - Vector-valued singular integrals and the $H^{1}-B M O$ duality, in: Probability Theory and Harmonic Analysis, J.-A. Chao and W. A. Woyczyński (eds.), Monographs and Textbooks in Pure and Appl. Math. 98, Marcel Dekker, New York, 1986, 1-19.

[11] D. L. Burkholder, A geometric condition that implies the existence of certain singular integrals of Banach-space-valued functions, in: Proc. Conf. in honor of A. Zygmund (Chicago, 1981), W. Beckner et al. (eds.), Wadsworth, Belmont, Calif., 1983, 270-286.

[12] M. L. Cartwright, Manuscripts of Hardy, Littlewood, Marcel Riesz and Titchmarsh, Bull. London Math. Soc. 14 (1982), 472-532.

[13] R. R. Coifman and G. Weiss, Transference Methods in Analysis, CBMS Regional Conf. Ser. in Math. 31, Amer. Math. Soc., Providence, 1977.

[14] I. Doust, Well-bounded operators and the geometry of Banach spaces, Thesis, University of Edinburgh, 1988.

[15] I. Doust and B. Z. Qiu, The spectral theorem for well-bounded operators, J. Austral. Math. Soc. Ser. A 54 (1993), 334-351.

[16] H. R. Dowson, Spectral Theory of Linear Operators, London Math. Soc. Monographs 12, Academic Press, London, 1978.

[17] H. R. Dowson and P. G. Spain, An example in the theory of well-bounded operators, Proc. Amer. Math. Soc. 32 (1972), 205-208.

[18] T. A. Gillespie, Logarithms of $L^{p}$ translations, Indiana Univ. Math. J. 24 (1975), $1037-1045$.

[19] - A spectral theorem for $L^{p}$ translations, J. London Math. Soc. (2) 11 (1975), 499-508.

[20] R. Hunt, B. Muckenhoupt and R. Wheeden, Weighted norm inequalities for the conjugate function and Hilbert transform, Trans. Amer. Math. Soc. 176 (1973), 227-251.

[21] J. R. Ringrose, On well-bounded operators, J. Austral. Math. Soc. 1 (1960), 334-343.

[22] -, On well-bounded operators II, Proc. London Math. Soc. (3) 13 (1963), 613-638.

[23] D. R. Smart, Conditionally convergent spectral expansions, J. Austral. Math. Soc. 1 (1960), 319-333.

[24] S. B. Stečkin, On bilinear forms, Dokl. Akad. Nauk SSSR 71 (1950), 237-240 (in Russian).

[25] E. C. Titchmarsh, Reciprocal formulae involving series and integrals, Math. Z. 25 (1926), 321-347.

[26] A. Zygmund, Trigonometric Series, Vol. I, Cambridge Univ. Press, Cambridge, 1959. 